\title{
A narrative review of the role of fibroblasts in the growth and development of neurogenic tumors
}

\author{
Cheng-Jiang Wei", Yi-Hui Gu\#, Wei Wang", Jie-Yi Ren, Xi-Wei Cui, Xiang Lian, Jin Liu, Hui-Jing Wang, \\ Bin Gu, Qing-Feng Li, Zhi-Chao Wang
}

Department of Plastic and Reconstructive Surgery, Shanghai Ninth People's Hospital, Shanghai Jiao Tong University School of Medicine, Shanghai, China

Contributions: (I) Conception and design: CJ Wei, YH Gu, W Wang, ZC Wang, QF Li; (II) Administrative support: QF Li, ZC Wang; (III) Provision of study materials or patients: CJ Wei, JY Ren, XW Cui, X Lian; (IV) Collection and assembly of data: YH Gu, X Lian, J Liu, HJ Wang; (V) Data analysis and interpretation: CJ Wei, W Wang, B Gu; (VI) Manuscript writing: All authors; (VII) Final approval of manuscript: All authors.

\#These authors contributed equally to this work.

Correspondence to: Qing-Feng Li, MD, PhD. Department of Plastic and Reconstructive Surgery, Shanghai Ninth People's Hospital, Shanghai Jiao Tong University School of Medicine, 639 Zhizaoju Road, Shanghai 200011, China. Email: liqingfeng@shsmu.edu.cn; dr_liqingfeng9@163.com; Zhi-Chao Wang, MD, MPH. Department of Plastic and Reconstructive Surgery, Shanghai Ninth People's Hospital, Shanghai Jiao Tong University School of Medicine, 639 Zhizaoju Road, Shanghai 200011, China. Email: shmuwzc@163.com; dr_wangzhichao@163.com.

\begin{abstract}
Neurogenic tumors, a group of tumors arising from neurogenic elements, could theoretically appear in every region of human bodies wherever nerves exist. Patients with these tumors suffer from both physical and psychological problems. However, as a relatively rare tumor type, therapies are relatively scarce for these tumors due to the limited understanding of the underlying mechanisms. Recently, a tailored tumor microenvironment containing multiple types of nonneoplastic cells has been considered to play an essential role in tumor survival, growth, and metastasis. Fibroblasts are a crucial constituent of the tumor microenvironment and have been found to promote tumor growth via multiple mechanisms. However, the understanding of the pivotal role of fibroblasts in the tumorigenesis and development of the neurogenic tumors is still incomplete, and studies in this area show differences in rates of progression among different neurogenic tumor subtypes. Nevertheless, all these neural crest-originated neoplasms show some similarities in the tumor microenvironment, indicating that studies of one subtype of neurogenic tumor might assist in clarifying the underlying mechanisms of other subtypes. This review aims to provide current studies showing the impacts of fibroblasts on major benign/malignant subtypes of neurogenic tumors, including neurofibromatosis type 1, neuroblastomas, pheochromocytomas, and malignant peripheral nerve sheath tumors. Multiple related mechanisms such as the fibroblasts regulating the tumor inflammation, angiogenesis, metabolism, and microenvironment establishment have been studied up to present. Consistently, we focus on how studies on various subtypes of these neurogenic tumors contribute to the establishment of potential future directions for further studies in this area. Clarifying the underlying mechanisms by which fibroblasts promote the growth and metastasis of neurogenic tumors will indicate new therapeutic targets for further clinical treatment.
\end{abstract}

Keywords: Fibroblasts; neurogenic tumors; tumor microenvironment

Submitted Apr 09, 2020. Accepted for publication Sep 18, 2020.

doi: $10.21037 /$ atm-20-3218

View this article at: http://dx.doi.org/10.21037/atm-20-3218 


\section{Introduction}

Neurogenic tumors, arising from any neurogenic elements, account for approximately $10-12 \%$ of all benign soft tissue tumors and $7-8 \%$ of all malignant soft tissues (1). These kinds of tumors are the most common causes of posterior mediastinal masses and could theoretically occur in the whole body wherever nerves exist $(2,3)$. The majority of these neoplasms can be divided into 3 types by origin: (I) nerve sheath tumors, such as neurofibromas, schwannomas, and malignant peripheral nerve sheath tumors (MPNSTs); (II) ganglion cell tumors, including ganglioneuromas and neuroblastomas; and (III) paraganglion cell tumors, including paragangliomas and pheochromocytomas (Pheos) (4). Surgical resection is considered in the treatment of the majority of these tumors (5), but both high recurrence rates in some subtypes, such as neurofibromatosis type 1 (NF1), and a proclivity for distant metastasis in a few malignant types suggest an urgent and continuing need for new medical treatment. A better understanding of the mechanisms of neurogenic tumor survival, growth, and metastasis is required to develop potential therapies in clinical treatment.

Interactions between the host stroma and tumor cells have recently been recognized as essential in tumor initiation and progression (6). One of the dominant components of the host microenvironment is the fibroblasts, and their role in tumor growth and development has been identified in multiple types of cancers, including breast cancer (7), liver cancer (8), and lung cancer (9). These activated fibroblasts are identified predominantly by the expression of $\alpha$-smooth muscle actin ( $\alpha$-SMA), which was first described in wound healing (10). Studies of the fibroblast functions in promoting cancers at present reveal multiple underlying mechanisms, including their role in promoting tumorigenesis and neoplasm metabolism, enhancing tumor metastasis and tumor angiogenesis, and assisting in the establishment of a cancer inflammatory microenvironment (10). In addition, fibroblasts are also differentiated from the neural crest (11), indicating that there might be a more complex and intriguing role of fibroblasts in neurogenic tumors. Recently, potential antitumor therapies directly targeting different aspects of fibroblasts or their secretory products, including fibroblast activating protein (FAP) (12), vascular endothelial growth factor (VEGF) (13), and hepatocyte growth factor (HGF), have also been studied in other tumors (14). For example, bevacizumab, a drug that inhibits VEGF, significantly increased patient overall survival in metastatic colorectal cancer, advanced cervical cancer, and non-small cell lung cancers (15-17). Consistent with this, cancer-associated fibroblasts (CAFs) are also implicated as essential in tumor therapy resistance via multiple mechanisms such as supporting residual tumor growth in melanoma and promoting cancer stem cell survival in breast cancers $(18,19)$.

As neurogenic tumors are a relatively rare type of cancer, studies aiming to clarify the exact role of fibroblasts in these tumors remain at the primary stages. However, the neurogenic tumors all originate from different elements of nerves, indicating possible similarities in the tumor microenvironment such as the existence of Schwann cells in the stroma. In this article, we will review the current findings on the importance of fibroblasts in the growth and development of neurogenic tumors. Moreover, we focus on discussing the possible universalities of the functions of fibroblasts in different subtypes of neurogenic tumors and offer further directions for research on this subject. A further understanding of the underlying mechanism of the complicated effects of fibroblasts on neurogenic tumors might indicate new therapeutic targets or strategies to reduce resistance to therapies. We present the following article with the Narrative Review reporting checklist (available at http://dx.doi.org/10.21037/atm-20-3218).

\section{Discussion}

\section{Fibroblasts play a role in the tumor immune microenvironment and assist microenvironment inflammation}

An inflammatory microenvironment has been found to be a hallmark of cancer (20). Neuroblastoma, arising from ganglion cells from the sympathoadrenal lineage of the neural crest, is the most common extracranial solid tumor in children (21). Larsson et al. demonstrated that a high-risk neuroblastoma group showed high microsomal prostaglandin E synthase-1 (mPGES-1) expression in the stroma, and these mPGES-1-positive cells also expressed one or several CAF markers (22). The mPGES-1 assist the formation of prostaglandin $\mathrm{E}_{2}\left(\mathrm{PGE}_{2}\right)$, which is a proinflammatory lipid mediator found in multiple types of cancers (23). A recent study by this team further described a significant decrease in tumor growth and migration ability after the usage of pharmacological mPGES-1 in preclinical models (24). Both findings suggest that the activated mPGES-1/PGE 2 pathway plays an essential role in the process of how fibroblasts promote the growth and 
development of neuroblastoma, and this pathway also have great potential for future therapy strategies. Furthermore, another study described the phenomenon that conditioned medium from neuroblastoma cells induced cancer associated fibroblast (CAF) marker expression by human bone marrow-derived mesenchymal stem cells (BM-MSCs), indicating the ability of tumor cells to promote fibroblast collaboration and activation (25). All these studies imply a possible bilateral interaction between fibroblasts and neuroblastoma cells in the generation of an inflammatory microenvironment.

Pheos, originating from the paraganglia from the neural crest, are another type of neurogenic tumor and have an incidence of approximately 2 to 8 per million people worldwide (26). A study of insulin-like growth factor 1 (IGF1), a critical factor in Pheo cells, described a decrease in the tumor cell proliferation rate under the treatment of conditioned medium from IGF1-haploinsufficient fibroblasts compared with that from wide-type fibroblasts (27). However, the tumor growth curve based on tumor volume did not show significant differences between these IGF1-haploinsufficient mouse group (L/n group) and the wide-type group (L/L group) despite the $100 \%$ tumor development rates in the $\mathrm{L} / \mathrm{L}$ group (while only $12.5 \%$ of mice in the $\mathrm{L} / \mathrm{n}$ group remained tumor free) (27). These controversial results indicated that the IGF1 mutation in fibroblasts is an impediment to tumor establishment on the first contact with the local microenvironment, but once these tumor cells survive at a specific location, tumor growth and development are hardly prevented by these fibroblasts. The underlying mechanisms of this phenomenon remain unknown, but the IGF-1 axis has been found to contribute to an immunosuppressive tumor microenvironment in multiple types of cancers (28). Whether there are similar underlying immunosuppression-related mechanisms or other possible processes merits further clarification.

Recently, there has been growing appreciation of the ability of fibroblasts to remodel the tumor immune microenvironment and inflammation (29). Current studies in neuroblastoma and Pheos have shown the potential of some classical inflammation pathways in promoting neurogenic tumorigenesis and tumor development by fibroblasts, which merits further confirmation in future studies. Moreover, a complete inflammatory process requires the participation of multiple types of stromal immune cells, and further studies are needed to clarify the complex correlations among these immune cells and fibroblasts.

\section{Activated fibroblasts are correlated with increased tumor metabolism}

Approximately $40 \%$ of Pheos are related to mutations of genes in one out of more than 30 suspected genes (26), and these genes include those encoding the succinate dehydrogenase subunits A-D (SDHA-D). Of these four subunits, $S D H B$ was first found to be highly related to tumor malignancy (30). A previous study on neuroblastoma showed a significant decrease in glucose uptake, an increase in lactate uptake, and a $92 \%$ increase in proliferation after coculture with human primary fibroblasts in these $S D H B$ silenced tumor cells compared with cells that cultured alone (31). Consistent with this, these $S D H B$-silenced cells were shown to obviously increase glucose uptake in fibroblasts, indicating the effects of these tumor cells on fibroblast metabolism (31). A later study in Pheos used a spheroids coculture system and demonstrated that fibroblast, activated by cocultured tumor cells, induced at least a 4-fold increase in $S D H B$-silenced Pheo cell migrations (32). Consistently, these activated fibroblasts cocultured with tumor cells released significantly more lactate than single-cultured fibroblasts, and lactate alone was found to promote tumor cell motility (32). Another study showed that primary fibroblasts coculture could reverse the decrease in cell growth caused by $S D H B$ knockdown (33). Furthermore, decreases in ATP and metabolites of central carbon metabolism were observed in cocultured $S D H B$-silenced cells, implicating a possible mechanism by which fibroblasts promoted proliferation and invasion of tumor cell via contributing to the mobilization of ATP and metabolic resources necessary for these processes (33).

Taken together, these studies indicated that fibroblasts might exert impacts on the metabolic processes of tumor cells. Consistent with this, similar mechanisms found both in neuroblastoma and Pheo indicate possible similarities in these neurogenic tumors. Further studies in one type of neurogenic tumor might also contribute to the understanding of the underlying mechanisms of other neurogenic tumor types, and awareness of this will significantly reduce research difficulties and accelerate the study process.

\section{Activated fibroblasts promote microvascular proliferation, which is inbibited by stromal Schwann cells}

At first, the number of Schwann cells in the stroma 
was found to be strongly related to neuroblastoma prognosis (34). Consistent with this, studies suggested that a higher level of Schwann cells in the stroma inhibited angiogenesis by secreting soluble factors (35). Later, a study analyzed 60 primary neuroblastoma tumors and revealed that Schwannian stroma-poor histopathology neuroblastomas showed increased amounts of CAFs with alpha $\alpha$-SMA expression and high microvascular proliferation compared with neoplasms with Schwannian stroma-rich histopathology (36). In addition, the study further revealed a sevenfold decrease in the number of $\alpha$-SMA-positive cells in neuroblastoma xenograft models with Schwann cells infiltration compared to controls (36), and this finding was in accordance with a previous study showing that neuroblastoma xenograft angiogenesis was inhibited by murine Schwann cells (37). These results just suggest that fibroblasts in the stroma might promote vascular proliferation in neuroblastoma and that this potential is impeded by Schwann cells. Further studies are needed to clarify the underlying mechanisms accounting for the microvascular proliferation in neuroblastoma potentiated by fibroblasts. Likewise, the interactions between fibroblasts and Schwann cells should also be considered.

Moreover, as neurogenic tumors all originate from nerves, Schwann cells are a nonnegligible part of the tumor microenvironment of these tumors. In other words, the interaction between stromal fibroblasts and Schwann cells is a common phenomenon in all neurogenic tumors. Further studies in understanding the role of fibroblasts in neurogenic tumors should take into consideration the possible impact of stromal Schwann cells.

\section{Activated mast cells stimulate mutated fibroblasts secreting collagens in NF1}

NF1 is a common autosomal dominant genetic disorder caused by mutation of the NF1 suppressor gene and affects approximately 1 in 3,000 individuals worldwide (38). Most NF1 patients are predisposed to benign peripheral nerve sheath tumors arising from Schwann cells, including cutaneous neurofibroma (CNF) and plexiform neurofibroma (PNF) (39). Fibroblasts have long been observed in the neurofibroma microenvironment, and there even studies in early stages to clarify whether the neurofibroma originated from fibroblasts $(40,41)$. Although the tumor cells are derived from Schwann cells, a large number of fibroblasts in the stroma implicate possible essential role of fibroblasts in tumorigenesis and development. Mast cells were first found recruited by a large amount of stem cell factors (SCFs) secreted by $\mathrm{Nf1}^{-/-}$Schwann cells to the NF1 microenvironment (42). In an autosomal dominant genetic disorder such as NF1, mutations of the NF1 gene occur in nonneoplastic cells, including mast cells. The $N f 1^{+/-}$mast cells were found far more sensitive to SCF than normal mast cells (42). Compared with normal mast cells, SCFstimulated $\mathrm{Nf1}^{+/-}$mast cells secreted 2.5 -fold more TGF- $\beta$ and potentiated $\mathrm{Nf}^{+/-}$fibroblast functions as shown by additional studies $(43,44)$. These stimulated fibroblasts secreted a large amount of collagen, which accounted for approximately half of the dry weight of the neurofibroma $(43,44)$. Notably, these mutated fibroblasts were also far more sensitive than normal fibroblasts, and this was found to result from the increased activity of c-abl secondary to increased Ras-GTP related to the NF1 gene (45).

Taken together, these studies show that the fibroblasts in NF1 are related to collagen secretion, and the stimulation of fibroblasts required the participation of both tumor cells and mast cells. Consistent with this, the impact of mutations in NF1 genes on stromal cells, especially fibroblasts, is essential in NF1 genesis and development. These findings should also be considered in the design of future NF1related studies.

\section{Studies in MPNSTs indicate possible homology between tumor cells and fibroblasts}

MPNSTs are some of the most common nonrhabdomyosarcoma soft-tissue sarcomas, and approximately $50 \%$ of MPNSTs arise in NF1 patients $(46,47)$. As they are derived from Schwann cells, MPNSTs express the S100 protein, a regular marker for Schwann cells (48). However, several case reports have shown that some tumor cells in some MPNST patients are positive for CD34, a marker of subpopulations of fibroblasts, indicating possible fibroblastic differentiations in MPNSTs (49,50). A recent study showed a possible association between CD34 positivity and neurotrophic receptor tyrosine kinase (NTRK) gene expression, indicating the possibility of therapeutic methods targeting NTRK in fibroblastic MPNSTs (51). However, studies in this area are rare, and the existing case analyses are not convincing enough. Further studies are needed to find the underlying mechanisms of this phenomenon, and whether these fibroblastic MPNSTs show specific clinicopathological features merits further investigation. 
Schwann cell precursors (SCPs), a type of neural crestderived cell, have recently been recognized as multipotent embryonic progenitors and have the potential to generate multiple cell types, including mature Schwann cells and fibroblasts $(52,53)$. The coexpression of fibroblast markers and Schwann cell markers in MPNSTs might be introduced by a shared precursor cell. Consistent with this, another study revealed that chromaffin cells, from which Pheo originates, were also derived from SCPs during murine embryo development (54). All these studies indicate high homology between fibroblasts and neurogenic tumor cells, suggesting an intriguing and critical relationship between these tumors and fibroblasts. Understanding the pivotal role and underlying mechanisms of fibroblasts in neurogenic tumors might also contribute to the understanding of the tumorigenesis from SCPs.

\section{Conclusions}

Studies clarifying the essential role of fibroblasts in multiple types of cancers have showed exciting results, but research surrounding fibroblasts in neurogenic tumors remained at the initial stage. In addition to neurogenic tumors being a relatively rare type of tumor, another important reason is the scarcity of evidence that fibroblasts are as "activated" in these types of tumors as they are in other cancers. For example, there is no report showing that NF1 fibroblasts express $\alpha$-SMA, an essential marker of most CAFs (10). Actually, multiple markers have been studied in CAFs such as $\alpha$-SMA and fibroblasts-activated protein (FAP), and the various markers divided these fibroblasts into different subtypes, which further indicated possible differences in functions (14). For example, FAP positive fibroblasts were found playing roles in bone and fat homeostasis (55). However, rare studies have shown the fibroblasts in neurogenic tumors positive for these markers. In order to emphasize the specificity of fibroblasts in neurogenic tumors compared with other types of tumors, we didn't use CAFs in this manuscript unless several referred studies used this term to particularly describe the differences of these fibroblasts compared with fibroblasts from normal tissues. A possible reason for this phenomenon is that most of the types of neurogenic tumors, such as NF1 and Pheo, are benign peripheral tumors, and the low level of malignancy contributes to a relatively low activated state of fibroblasts. Similar circumstances were also described in other benign tumors, such as breast fibroadenoma, in which only 3 of 11 cases were showed $\alpha$-SMA in $50 \%$ of stromal cells compared with 11 breast carcinoma cases that had $\alpha$-SMA positivity in $80 \%$ of stromal cells (56). This finding suggests that a low malignant level might be a possible explanation for why fibroblasts in some neurogenic tumors don't express "activated" markers such as $\alpha$-SMA. However, studies in wound healing and other cancers indicated the activation and expression of $\alpha$-SMA were induced by TGF- $\beta(10,57)$, and the 2.5 -fold increase in TGF- $\beta$ secretion in NF1 implied that there might be other underlying mechanisms behind this phenomenon. Studies in neuroblastoma present a possible explanation: fibroblasts activation might be inhibited by Schwann cells, which merits further investigation in future studies.

Consistently, previous studies of the fibroblasts effect in different subtypes of neurogenic tumors had unequal rates of progression and various directions (Table 1, Figure 1). Nevertheless, these neurogenic neoplasms all originate from the neural crest, indicating possible homogeneity in tumorigenesis and a similar tumor growth microenvironment. In other words, studies of fibroblasts in one subtype of these tumors contribute to the understanding and further study design in all kinds of neurogenic tumor subtypes. This review provides an update on current studies showing multiple mechanisms by which fibroblasts promote different subtypes of neurogenic tumors. Considering the evidence, we recommend the following aspects be taken into account in future studies: (I) gene mutations in fibroblasts need to be considered. In autosomal genetic disorders such as NF1, mutations in NF1 genes occur in nonneoplastic cells, including fibroblasts, in the microenvironment, and these mutated cells show distinct characteristics compared with normal fibroblasts. Consistently, mutated fibroblasts have also been found to be essential in tumorigenesis and tumor development in other subtypes of cancer, such as Pheos. Abnormalities in fibroblasts imply more profound and complex impacts on neurogenic tumors, which merits further investigation. (II) The interactions with Schwann cells need to be considered. Schwann cells, the peripheral nerve glial cells, can be found in all kinds of neurogenic neoplasms, serving cooperatively with stromal cells or even just as the origin of tumor cells. Fibroblasts in neurogenic tumors have rarely been reported to express activation markers such as $\alpha$-SMA despite the presence of multiple stimulators including TGF- $\beta$, and studies in neuroblastoma suggested a possible explanation: the activation of fibroblasts might be impeded by Schwann cells. (III) The impact of tumor cells needs to be considered. Studies of fibroblasts in tumors have indicated the contact 
Table 1 The role of fibroblasts in different types of neurogenic tumors

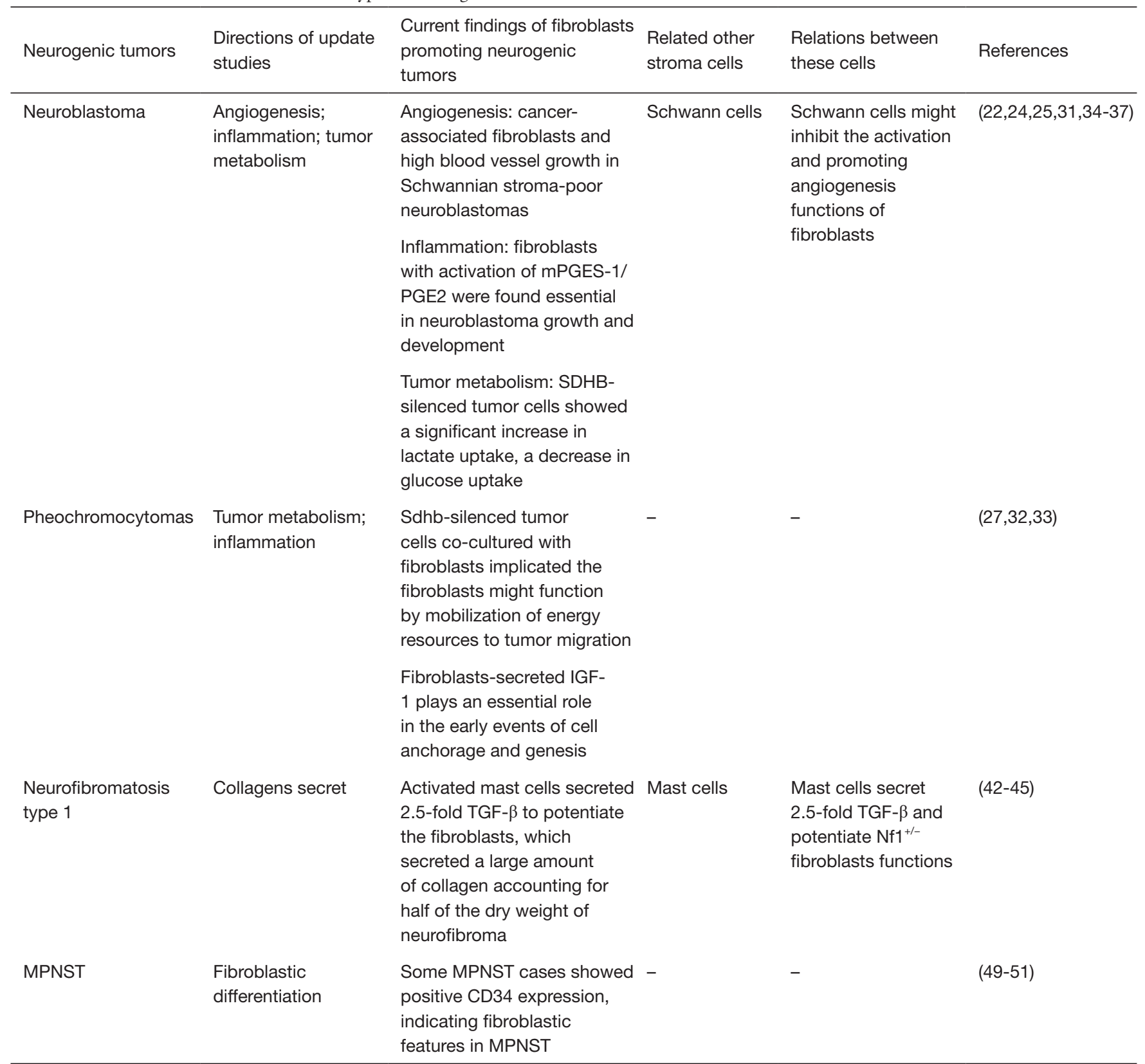

mPGES-1/PGE2, microsomal prostaglandin E synthase-1/prostaglandin E2; SDHB, succinate dehydrogenase subunits B; IGF-1, insulinlike growth factor 1 ; TGF- $\beta$, transforming growth factor $\beta$; MPNST, malignant peripheral nerve sheath tumors; CD34, cell differentiation factor 34 . 


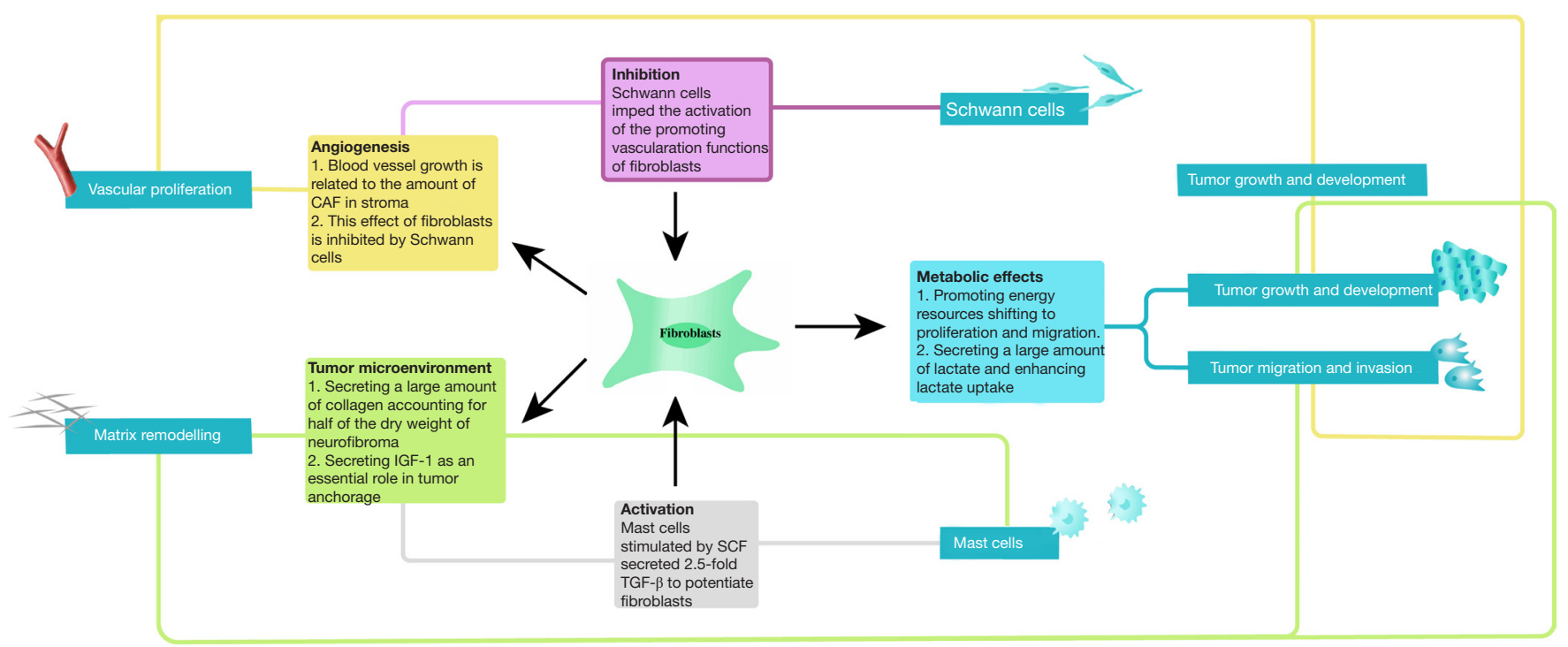

Figure 1 Summary of fibroblast functions in neurogenic tumors and the underlying mechanisms. Fibroblasts play an essential role in neurogenic tumors growth and development by various mechanisms. CAF, cancer-associated fibroblasts; IGF, insulin-like growth factor 1; SCF, stem cell factor; TGF- $\beta$, transforming growth factor $\beta$.

between cancer cells and fibroblasts can promote the activation of fibroblasts in multiple ways $(55,58)$, and this has also been shown in studies in Pheos mentioned above.

Although studies in this area are rare at the primary stage, current data have implicated the essential role of fibroblasts in neurogenic tumor development. At present, clinical trials targeting fibroblasts in neurogenic tumors are rare because of the clarification of the underlying mechanisms. But with an understanding of the mechanisms in place, we are confident about the prospect of new fibroblast-targeted therapies in the treatment of neurogenic tumors.

\section{Acknowledgments}

Funding: This work was supported by grants from Youth Doctor Collaborative Innovation Team Project (QC201803) of Shanghai Ninth People's Hospital, Shanghai Jiao Tong University School of Medicine; the Project of Biobank (No. YBKA201901) from Shanghai Ninth People's Hospital, Shanghai Jiao Tong University School of Medicine; the Shanghai Youth Top-Notch Talent Program (201809004); “Chenguang Program" supported by Shanghai Education Development Foundation and Shanghai Municipal Education Commission (19CG18) and Science and Technology Commission of Shanghai
Municipality (19JC1413), Shanghai Rising-Star Program (20QA1405600).

\section{Footnote}

Reporting Checklist: The authors have completed the Narrative Review reporting checklist. Available at http:// dx.doi.org/10.21037/atm-20-3218

Conflicts of Interest: All authors have completed the ICMJE uniform disclosure form (available at http://dx.doi. org/10.21037/atm-20-3218). ZCW serves as an unpaid Section Editor of Annals of Translational Medicine from Oct 2019 to Sep 2020. WW serves as an unpaid Section Editor of Annals of Translational Medicine from Oct 2019 to Sep 2020. The other authors have no conflicts of interest to declare.

Ethical Statement: The authors are accountable for all aspects of the work in ensuring that questions related to the accuracy or integrity of any part of the work are appropriately investigated and resolved.

Open Access Statement: This is an Open Access article distributed in accordance with the Creative Commons Attribution-NonCommercial-NoDerivs 4.0 International 
License (CC BY-NC-ND 4.0), which permits the noncommercial replication and distribution of the article with the strict proviso that no changes or edits are made and the original work is properly cited (including links to both the formal publication through the relevant DOI and the license). See: https://creativecommons.org/licenses/by-nc-nd/4.0/.

\section{References}

1. Newton HB. editor. Handbook of neuro-oncology neuroimaging. 2nd edition. Amsterdam; Boston: Elsevier/ Academic Press, 2016. xxi, 841 pages.

2. Pretorius ES, Solomon JA. Radiology secrets plus. 3rd edition. Secrets series. Philadelphia, PA: Mosby/Elsevier, 2011. xv, 562 pages.

3. Cardillo G, Carleo F, Khalil MW, et al. Surgical treatment of benign neurogenic tumours of the mediastinum: a single institution report. Eur J Cardiothorac Surg 2008;34:1210-4.

4. Reed JC, Hallet KK, Feigin DS. Neural tumors of the thorax: subject review from the AFIP. Radiology 1978;126:9-17.

5. Ratbi MB, El Oueriachi F, Arsalane A, et al. Surgery of benign neurogenic tumors in adults: single institution experience. Pan Afr Med J 2014;19:288.

6. Schulz M, Salamero-Boix A, Niesel K, et al. Microenvironmental Regulation of Tumor Progression and Therapeutic Response in Brain Metastasis. Front Immunol 2019;10:1713.

7. Houthuijzen JM, Jonkers J. Cancer-associated fibroblasts as key regulators of the breast cancer tumor microenvironment. Cancer Metastasis Rev 2018;37:577-97.

8. Affo S, Yu LX, Schwabe RF. The Role of CancerAssociated Fibroblasts and Fibrosis in Liver Cancer. Annu Rev Pathol 2017;12:153-86.

9. Mahale J, Smagurauskaite G, Brown K, et al. The role of stromal fibroblasts in lung carcinogenesis: A target for chemoprevention? Int J Cancer 2016;138:30-44.

10. Kalluri R. The biology and function of fibroblasts in cancer. Nat Rev Cancer 2016;16:582-98.

11. Mayor R, Theveneau E. The neural crest. Development 2013;140:2247-51.

12. Wuest T, Moosmayer D, Pfizenmaier K. Construction of a bispecific single chain antibody for recruitment of cytotoxic $T$ cells to the tumour stroma associated antigen fibroblast activation protein. J Biotechnol 2001;92:159-68.

13. Morrow PK, Murthy RK, Ensor JD, et al. An open-label, phase 2 trial of RPI.4610 (Angiozyme) in the treatment of metastatic breast cancer. Cancer 2012;118:4098-104.

14. Khan GJ, Sun L, Khan S, et al. Versatility of Cancer Associated Fibroblasts: Commendable Targets for Antitumor Therapy. Curr Drug Targets 2018;19:1573-88.

15. Bennouna J, Sastre J, Arnold D, et al. Continuation of bevacizumab after first progression in metastatic colorectal cancer (ML18147): a randomised phase 3 trial. Lancet Oncol 2013;14:29-37.

16. Socinski MA, Jotte RM, Cappuzzo F, et al. Atezolizumab for First-Line Treatment of Metastatic Nonsquamous NSCLC. N Engl J Med 2018;378:2288-301.

17. Tewari KS, Sill MW, Long HJ, 3rd, et al. Improved survival with bevacizumab in advanced cervical cancer. $\mathrm{N}$ Engl J Med 2014;370:734-43.

18. Hirata E, Girotti MR, Viros A, et al. Intravital imaging reveals how BRAF inhibition generates drug-tolerant microenvironments with high integrin beta1/FAK signaling. Cancer Cell 2015;27:574-88.

19. Su S, Chen J, Yao H, et al. CD10+GPR77+ CancerAssociated Fibroblasts Promote Cancer Formation and Chemoresistance by Sustaining Cancer Stemness. Cell 2018;172:841-856.e16.

20. Hanahan D, Weinberg RA. Hallmarks of cancer: the next generation. Cell 2011;144:646-74.

21. Louis CU, Shohet JM. Neuroblastoma: molecular pathogenesis and therapy. Annu Rev Med 2015;66:49-63.

22. Larsson K, Kock A, Idborg H, et al. COX/mPGES-1/ PGE2 pathway depicts an inflammatory-dependent highrisk neuroblastoma subset. Proc Natl Acad Sci U S A 2015;112:8070-5.

23. Nakanishi M, Rosenberg DW. Multifaceted roles of PGE2 in inflammation and cancer. Semin Immunopathol 2013;35:123-37.

24. Kock A, Larsson K, Bergqvist F, et al. Inhibition of Microsomal Prostaglandin E Synthase-1 in CancerAssociated Fibroblasts Suppresses Neuroblastoma Tumor Growth. EBioMedicine 2018;32:84-92.

25. Hashimoto O, Yoshida M, Koma Y, et al. Collaboration of cancer-associated fibroblasts and tumour-associated macrophages for neuroblastoma development. J Pathol 2016;240:211-23.

26. Zhikrivetskaya SO, Snezhkina AV, Zaretsky AR, et al. Molecular markers of paragangliomas/ pheochromocytomas. Oncotarget 2017;8:25756-82.

27. Martin A, Venara M, Matho C, et al. Fibroblast deficiency of insulin-like growth factor 1 receptor type 1 (IGF1R) impairs initial steps of murine pheochromocytoma development. Biochimie 2019;163:108-16. 
28. Yahya MA, Sharon SM, Hantisteanu S, et al. The Role of the Insulin-Like Growth Factor 1 Pathway in Immune Tumor Microenvironment and Its Clinical Ramifications in Gynecologic Malignancies. Front Endocrinol (Lausanne) 2018;9:297.

29. Lakins MA, Ghorani E, Munir H, et al. Cancer-associated fibroblasts induce antigen-specific deletion of CD8 (+) T Cells to protect tumour cells. Nat Commun 2018;9:948.

30. Baysal BE, Maher ER. 15 years of paraganglioma: Genetics and mechanism of pheochromocytoma-paraganglioma syndromes characterized by germline SDHB and SDHD mutations. Endocr Relat Cancer 2015;22:T71-82.

31. Rapizzi E, Fucci R, Giannoni E, et al. Role of microenvironment on neuroblastoma SK-N-AS SDHBsilenced cell metabolism and function. Endocr Relat Cancer 2015;22:409-17.

32. D'Antongiovanni V, Martinelli S, Richter S, et al. The microenvironment induces collective migration in SDHBsilenced mouse pheochromocytoma spheroids. Endocr Relat Cancer 2017;24:555-64.

33. Richter S, D'Antongiovanni V, Martinelli S, et al. Primary fibroblast co-culture stimulates growth and metabolism in Sdhb-impaired mouse pheochromocytoma MTT cells. Cell Tissue Res 2018;374:473-85.

34. Shimada H, Ambros IM, Dehner LP, et al. The International Neuroblastoma Pathology classification (the Shimada system). Cancer 1999;86:364-72.

35. Crawford SE, Stellmach V, Ranalli M, et al. Pigment epithelium-derived factor (PEDF) in neuroblastoma: a multifunctional mediator of Schwann cell antitumor activity. J Cell Sci 2001;114:4421-8.

36. Zeine R, Salwen HR, Peddinti R, et al. Presence of cancer-associated fibroblasts inversely correlates with Schwannian stroma in neuroblastoma tumors. Mod Pathol 2009;22:950-8.

37. Liu S, Tian Y, Chlenski A, et al. Cross-talk between Schwann cells and neuroblasts influences the biology of neuroblastoma xenografts. Am J Pathol 2005;166:891-900.

38. Huson SM, Compston DA, Harper PS. A genetic study of von Recklinghausen neurofibromatosis in south east Wales. II. Guidelines for genetic counselling. J Med Genet 1989;26:712-21.

39. Zhu Y, Ghosh P, Charnay P, et al. Neurofibromas in NF1: Schwann cell origin and role of tumor environment. Science 2002;296:920-2.

40. Rosenbaum T, Boissy YL, Kombrinck K, et al. Neurofibromin-deficient fibroblasts fail to form perineurium in vitro. Development 1995;121:3583-92.
41. Kluwe L, Friedrich R, Mautner VF. Loss of NF1 allele in Schwann cells but not in fibroblasts derived from an NF1associated neurofibroma. Genes Chromosomes Cancer 1999;24:283-5.

42. Yang FC, Ingram DA, Chen S, et al. Neurofibromindeficient Schwann cells secrete a potent migratory stimulus for Nf1+/- mast cells. J Clin Invest 2003;112:1851-61.

43. Jaakkola S, Peltonen J, Riccardi V, et al. Type 1 neurofibromatosis: selective expression of extracellular matrix genes by Schwann cells, perineurial cells, and fibroblasts in mixed cultures. J Clin Invest 1989;84:253-61.

44. Yang FC, Chen S, Clegg T, et al. Nf1+/- mast cells induce neurofibroma like phenotypes through secreted TGF-beta signaling. Hum Mol Genet 2006;15:2421-37.

45. Staser K, Yang FC, Clapp DW. Mast cells and the neurofibroma microenvironment. Blood 2010;116:157-64.

46. Ducatman BS, Scheithauer BW, Piepgras DG, et al. Malignant peripheral nerve sheath tumors. A clinicopathologic study of 120 cases. Cancer 1986;57:2006-21.

47. Ferrari A, Miceli R, Rey A, et al. Non-metastatic unresected paediatric non-rhabdomyosarcoma soft tissue sarcomas: results of a pooled analysis from United States and European groups. Eur J Cancer 2011;47:724-31.

48. Miettinen MM, Antonescu CR, Fletcher CDM, et al. Histopathologic evaluation of atypical neurofibromatous tumors and their transformation into malignant peripheral nerve sheath tumor in patients with neurofibromatosis 1-a consensus overview. Hum Pathol 2017;67:1-10.

49. Houreih MA, Eyden B, Deolekar M, et al. A case of fibroblastic low-grade malignant peripheral nerve sheath tumor--a true neurofibrosarcoma. Ultrastruct Pathol 2007;31:347-56.

50. Mills AM, Karamchandani JR, Vogel H, et al. Endocervical fibroblastic malignant peripheral nerve sheath tumor (neurofibrosarcoma): report of a novel entity possibly related to endocervical CD34 fibrocytes. Am J Surg Pathol 2011;35:404-12.

51. Wells AE, Mallen AM, Bui MM, et al. NTRK-1 fusion in endocervical fibroblastic malignant peripheral nerve sheath tumor marking eligibility for larotrectinib therapy: A case report. Gynecol Oncol Rep 2019;28:141-4.

52. Kastriti ME, Adameyko I. Specification, plasticity and evolutionary origin of peripheral glial cells. Curr Opin Neurobiol 2017;47:196-202.

53. Furlan A, Adameyko I. Schwann cell precursor: a neural crest cell in disguise? Dev Biol 2018;444 Suppl 1:S25-35.

54. Furlan A, Dyachuk V, Kastriti ME, et al. Multipotent 


\section{Page 10 of 10}

peripheral glial cells generate neuroendocrine cells of the adrenal medulla. Science 2017;357.

55. Sahai E, Astsaturov I, Cukierman E, et al. A framework for advancing our understanding of cancer-associated fibroblasts. Nat Rev Cancer 2020;20:174-86.

56. Sappino AP, Skalli O, Jackson B, et al. Smooth-muscle differentiation in stromal cells of malignant and nonmalignant breast tissues. Int J Cancer 1988;41:707-12.

57. Ronnov-Jessen L, Petersen OW. Induction of alpha-

Cite this article as: Wei CJ, Gu YH, Wang W, Ren JY, Cui XW, Lian X, Liu J, Wang HJ, Gu B, Li QF, Wang ZC. A narrative review of the role of fibroblasts in the growth and development of neurogenic tumors. Ann Transl Med 2020;8(21):1462. doi: 10.21037/atm-20-3218 smooth muscle actin by transforming growth factor-beta 1 in quiescent human breast gland fibroblasts. Implications for myofibroblast generation in breast neoplasia. Lab Invest 1993;68:696-707.

58. Strell C, Paulsson J, Jin SB, et al. Impact of EpithelialStromal Interactions on Peritumoral Fibroblasts in Ductal Carcinoma in Situ. J Natl Cancer Inst 2019;111:983-95. 